\title{
Bettina Alavi
}

\section{Lernen Schüler/innen Geschichte im Digitalen anders?}

In der Geschichtsdidaktik sind empirische Untersuchungen zum Lernen mit digitalen Medien immer noch rar und finden sich sehr verstreut. ${ }^{1}$ Zur Beantwortung der Titelfrage „Lernen Schüler/innen Geschichte im Digitalen anders?“ gibt es bisher nur wenige Antworten. Deshalb wird in diesem Beitrag der Versuch unternommen, die vorhandenen Untersuchungen in einer Art Bestandsaufnahme zu systematisieren. Dazu wird zunächst anhand eines einführenden Beispiels die Relevanz solcher empirischer Forschungen verdeutlicht, dann die vorhandenen Untersuchungen zum einen mit Hilfe des Modells der vier Modi historischen Lernens im Digitalen sowie zum anderen bezugnehmend auf deren methodische Zugangsweisen systematisiert. Auf die pointierte Darstellung von einigen Ergebnissen dieser Forschungen folgt das Ausweisen dringend zu bearbeitender Desiderate.

\section{Einführendes Beispiel}

Der elfjährige Sohn einer Kollegin besuchte diese im Praktikumsamt unserer Hochschule und kommt dort in die Küche, die ein älteres Modell mit zusammengestückeltem Mobiliar enthält. Erstaunt ruft er aus: „Hier sieht es so aus wie bei Anne Frank!“

Für ein Referat über die Stadt Amsterdam im Fach Geographie hatte er die Seiten des Anne-Frank-Hauses in Amsterdam besucht, auf denen ein virtueller 3D-Rundgang durch das Hinterhaus angeboten wird, in dem sich die Familie Frank versteckt hatte. ${ }^{2}$ Die Ausstattung der Einzelräume hatte er sich offensichtlich gut gemerkt, werden die Räume doch auf den Webseiten ästhetisch ansprechend und mit von Schauspielern eingesprochenen Geschichten angereichert

1 Das gilt nicht nur für das Fach Geschichte, sondern beispielsweise auch für das Fach Deutsch, wo zwar der Einsatz digitaler Medien weiter verbreitet ist, empirische Studien darüber aber auch selten sind. Vgl. Thomas Möbius: Empirische Forschung zum Einsatz digitaler Medien im Deutschunterricht. In: Thomas Möbius/Volker Frederking/Axel Krommer (Hrsg.): Digitale Medien im Deutschunterricht. Hildesheim (erscheint voraussichtlich 2014). Der Autor hat mir dankenswerter Weise sein Manuskript vorab zur Verfügung gestellt.

2 Das Küchenbild, auf das er Bezug nahm, findet sich unter http://www.annefrank.org/de/ Subsites/Home/Betritt-das-3D-Haus/\#/house/17/ (aufgerufen am 22.7.2013). 
präsentiert. Aus seinem eigenen Lebensumfeld kannte der Schüler - worauf die Kollegin hinwies - keine älteren Küchen, sondern nur moderne Einbauküchen. Dies brachte ihn dazu, die heutige Küche des Praktikumsamtes mit der virtuell besuchten Küche der Franks in Amsterdam assoziativ zu verbinden. Dabei ist die Küche der Franks nur im virtuellen Rundgang zu sehen, denn im Museum selber sind die Räume leer, da die Möbel nach der Verhaftung der Versteckten abtransportiert wurden. Die im 3D-Rundgang präsentierten Räume orientieren sich an den Tagebuchbeschreibungen der Anne Frank.

An dieser Geschichte kann beispielhaft gezeigt werden, wie sich die Bedingungen und Auswirkungen des digitalen Wandels auf das historische Lernen, aber auch auf das Geschichtsbewusstsein und auf die Geschichts- und Erinnerungskultur gestalten.

\section{Multimedialität als Prägefaktor des Geschichtsbewusstseins}

Der 3D-Rundgang stellt eine rekonstruierte Visualisierung des Vergangenen dar, die auf der Basis einer schriftlichen Quelle - des Tagebuchs der Anne Frank - erstellt wurde. Diese Rekonstruktion ist im Internet weltweit schnell verfügbar und offensichtlich für die Benutzergruppe der heranwachsenden Schüler/innen äußerst wirkmächtig: Die lebensnahe und farbenfrohe Visualisierung, die eingesprochenen Geschichten aus dem Leben der versteckt Lebenden, die mit Bildern und dem Tagebuch angereicherte Kontextualisierung mit dem tragischen Schicksal eines gleichaltrigen Mädchens hat die Vorstellung des Jungen so nachhaltig geprägt, dass er sich viele Details gemerkt hat. Allein das Lesen des Tagebuchs hätte diese Wirkung wohl kaum erzielt. Vielmehr scheint das Zusammenspiel der Einzelelemente der multimedialen Präsentation, die mehrere Sinneseindrücke gleichzeitig anspricht (Hören, Sehen) und quellenmäßig Überliefertes in einer ästhetischen Umsetzung (eingesprochene Szenen, Rekonstruktion des Mobiliars) zur Verfügung stellt, die Adressatengruppe für das tragische biographische Schicksal eines gleichaltrigen Mädchens besonders anzusprechen.

\section{Informelles Lernen}

Die Vorstellung des genannten Elfjährigen von einer älteren Küche orientiert sich an diesem rekonstruierten historischen Beispiel, wobei die Prägung dieser Küchenvorstellung nicht das Ziel seiner Internetrecherche über Amsterdam war, innerhalb derer der Besuch des virtuellen Rundgangs quasi nur ein Neben- 
schauplatz darstellte. Offensichtlich hat er für sein Geographiereferat über Amsterdam nach einer Google-Suche auf mehreren Webseiten gesurft und war aus Interesse am Anne-Frank-Haus „hängengeblieben“. Wahrscheinlich hat er bei seiner Recherche noch mehr über Anne Frank gelernt, wobei es durchaus interessant wäre, weitere nicht intendierte Lerneffekte $\mathrm{zu}$ erfassen, weil dadurch Rückschlüsse auf den Aufbau von Geschichtsbewusstsein gezogen werden könnten. Denn gerade das Zusammenspiel von formellem historischem Lernen im Geschichtsunterricht und informellem Lernen im Internet scheint eine neue Dimension der interdependenten Verarbeitung von Geschichte $\mathrm{zu}$ eröffnen. Wo Spielfilme, Fernsehsendungen, Computerspiele schon einen solchen Weg angebahnt haben, erscheinen die Möglichkeiten virtueller Exkursionen, der fast unbegrenzten Informationsangebote im Internet sowie der schnellen elektronischen Kommunikation für das informelle Lernen von Geschichte viele neue Impulse zu geben. Das informelle Lernen kann dadurch individueller werden, gerade weil es im Internet zunehmend adressatengerechtere Angebote gibt, wie das Amsterdamer Beispiel zeigt. So könnte es sehr gewinnbringend sein, den Zusammenhang zwischen formellem und informellem historischen Lernen empirisch zu erforschen und die Auswirkungen auf das Geschichtsbewusstsein zu untersuchen, weil dadurch die Angebote und Bedingungen von Digitalität für das historische Lernen bewusster genutzt werden könnten.

\section{Globalisierung von Erinnerungskultur}

Diese kleine Geschichte macht auch deutlich, dass die Nutzung von Geschichtsangeboten durch das digitale Medium nicht nur individueller geworden ist, sondern auch internationaler. Der elfjährige Junge griff von Deutschland aus auf die Website des Anne-Frank-Hauses $\mathrm{zu}$, diese ist weltweit verfügbar und wird in mehreren Sprachversionen zur Verfügung gestellt. Hinzu kommt die Repräsentanz des Anne-Frank-Hauses bei Facebook, so dass die Schüler/innen mit einfachem und für sie lebensnahen Zugriff kommunizieren können. Schüler/innen aus aller Welt „besuchen“ so das Anne-Frank-Haus auch wenn eine Exkursion aus Kosten- oder aus Auslastungsgründen nicht möglich ist. ${ }^{3}$ So fragt sich, wie die Schüler/innen weltweit diese Website rezi-

\footnotetext{
3 Auf die Website wird auch deshalb so viel Wert gelegt, weil die Kapazität des Anne-FrankHauses, was reale Besucher/innen angeht, seit Jahren an der Grenze des Machbaren liegt. Nach Aussage von Ita Amahorseija und Gerrit Netten vom Anne-Frank-Haus Amsterdam auf der Tagung „httpasts://digitalmemoryonthenet“ der Bundeszentrale für politische Bildung vom 14.-16.4.2011 in Berlin sollen dadurch auch Besucherströme reduziert werden.
} 
pieren und worin interkulturelle Unterschiede bestehen könnten. Gerade bei der derzeitigen Tendenz der Universalisierung des Holocaust wäre die empirische Untersuchung des sich globalisierenden Geschichtsbewusstseins im Spannungsfeld interkultureller Unterschiede reizvoll, weil dadurch mehr über die Veränderungsprozesse des Geschichtsbewusstsein durch die digitalen Medien erfahrbar gemacht werden könnte.

Die Titelfrage meines Vortrags „Lernen Schüler/innen Geschichte im Digitalen anders?“ kann also so interpretiert werden, dass die digitalen Medien die historische Erkenntnisse wesentlich mit formen, die Zugänge zum historischen Lernen informeller und unberechenbarer geworden sind und es Ziel der Geschichtsdidaktik sein muss, die Wechselwirkung zwischen dem historischen Lernen und den Bedingungen der Digitalisierung stärker $\mathrm{zu}$ untersuchen insbesondere auch empirisch. Gleichzeitig kann hier schon die Titelfrage modifiziert werden, denn es ist offenbar, dass es nicht um anderes historisches Lernen geht, denn die Denkoperationen, die das historische Lernen bedingen (wie Analysieren, Kontextualisieren, Interpretieren, Datieren) scheinen gleich geblieben. Was sich aber verändert hat und die Erkenntnis von Geschichte wesentlich mitgestaltet sind die Bedingungen für das historische Lernen. Das Internet gleicht einem eigenen Kulturraum mit eigenen Regeln, Sprache und Kommunikationsverfahren, ${ }^{4}$ die Virtualität durchdringt zunehmend die Realität, so dass deren Grenzen verschwimmen. Die Titelfrage könnte also umformuliert werden in: Wie gestaltet sich historisches Lernen unter den Bedingungen der Digitalität?

Um die empirischen Forschungen systematisieren zu können, erscheint ein Theoriemodell zweckmäßig, das die Besonderheiten des historischen Lernens im digitalen Medium erfasst. Deshalb wird hier Bezug genommen auf die vier Modi des historischen Lernens im Digitalen, wie sie 2012 von Bernsen/König/Spahn formuliert wurden. ${ }^{5}$ Sie unterscheiden in Lernen an digitalen Medien, Lernen mit digitalen Medien, Lernen über digitale Medien und Lernen im digitalen Medium. Um das Theoriemodell besser veranschaulichen $\mathrm{zu}$ können, werden relevante Forschungsfragen aufgeführt, die sich inhaltlich auf das Einstiegsbeispiel der Website des Anne-Frank-Hauses beziehen. Dabei wird auch deutlich werden,

4 Darauf hat insbesondere Thomas Krüger in seinem Vortrag auf der dieser Publikation zugrunde liegenden Tagung hingewiesen.

5 Vgl. Daniel Bernsen/Alexander König/Thomas Spahn: Medien und historisches Lernen: Ein Verhältnisbestimmung und ein Plädoyer für eine digitale Geschichtsdidaktik. In: Zeitschrift für digitale Geschichtswissenschaften, 1/2012. http://universaar.uni-saarland.de/journals/ index.php/zdg/article/view/294 (aufgerufen 03.03.2013). Vgl. für die Ausführungen zu den Modi S. 17f. 
dass sich die einzelnen Modi nicht immer trennscharf unterscheiden lassen, sondern dass es Interdependenzen und Überlappungsbereiche gibt.

Lernen an digitalen Medien nimmt die Medien in ihrer Potentialität für das Lernen in den Fokus und berücksichtigt dabei sowohl intentionale als auch informelle Lernprozesse: Wie recherchieren Schüler/innen auf der Website des Anne-Frank-Hauses? Was merken sie sich? Welche informellen Lernprozesse sind damit verbunden? Wovon sind diese informellen Lernprozesse abhängig? Was merken sich argentinische, südafrikanische, israelische oder japanische Schüler/innen? Welche Vorstellungen integrieren sie wie in ihr Geschichtsbewusstsein? Wie funktionieren intentionale historische Lernprozesse im 3D-Rundgang? In welchen Kompetenzstufen erkennen und dekonstruieren die Schüler/innen den Konstruktcharakter des 3D-Rundgangs? Welche Rolle nimmt der 3D-Rundgang im Lernensemble von Tagebuch, Anne-Frank-Haus, Website und Geschichtsunterricht für das Lernen über Anne Frank als Beispiel für ein Schicksal im Nationalsozialismus ein?

Lernen mit digitalen Medien stellt den Werkzeugcharakter digitaler Medien in den Mittelpunkt und untersucht, wie Medien als Lern- und Denkwerkzeuge eingesetzt werden, um historisches Lernen zu unterstützen: Wie kommunizieren die Schüler/innen mittels digitaler Medien über Anne Frank, z.B. mit Hilfe von Facebook? Was geschieht, wenn sie in Blogs ihre Arbeitsergebnisse zu Anne Frank kommunizieren? Wie funktionieren kooperative historische Lernprozesse?

Lernen über digitale Medien bedeutet zum einen die Geschichte der Medien zu betrachten und zum anderen deskriptiv Aufbau, Funktion und Handhabung der einzelnen Medien in den Mittelpunkt zu stellen: Wie entsteht ein 3D-Rundgang zum Anne-Frank-Hinterhaus? Was steht genau so im Tagebuch? Was schreibt Anne Frank vielleicht nicht? Welche Lücken füllt die Visualisierung wie aus? Wie wirkt ein solcher 3D-Rundgang auf unterschiedliche Nutzer? Im 3D-Rundgang fehlen Gerüche und haptische Elemente, außerdem Sinneseindrücke durch Sonne/Helligkeit - zu welchen Erkenntnissen kann es deshalb nicht kommen? Inwiefern ist ein solcher 3D-Rundgang weltweit verständlich?

Lernen im digitalen Medium fokussiert auf die Nutzung des Mediums als Denk- und Lernraum und nimmt das medienspezifisch begrenzte Set an Funktionalitäten in den Blick, d.h. schaut nach deren Angemessenheit im Hinblick auf das Lernobjekt, den Prozess und das Produkt des historischen Lernens: Wie hat der Schüler seine Aufgabe gelöst, ein Referat über Amsterdam zu halten: Welche Recherchemedien nutzt er? Welche Recherchewege geht er? Welche Präsentationsform mit spezifischen Möglichkeiten und Grenzen wählt er? 
Ordnet man die vorhandenen empirischen Arbeiten zum historischen Lernen unter den Bedingungen der Digitalität mit ihren Forschungsfragen diesen Modi zu, so ergibt sich folgendes Bild:

Zum historischen Lernen an digitalen Medien liegen mehrere empirische Arbeiten vor: So wurde von Alavi $2007^{6}$ und Alavi/Schäfer 20107 untersucht, wie Schüler/innen mit Selbstlernsoftware lernen. Diese Selbstlernsoftware beinhaltet Aufgaben zu historischen Themen, die den Anspruch haben, ergänzend zum Geschichtsunterricht den Schüler/innen eine selbständige Bearbeitung zu gewährleisten. Hier werden Lern- und Kooperationsstrategien zur Lösung der „historischen“ Lernaufgabe, die Verzahnung mit Wissen und Methodenkenntnissen aus dem Geschichtsunterricht, die beobachteten Schwierigkeiten und die Lerneffekte untersucht. ${ }^{8}$ Auch die empirischen Untersuchungen von Danker/Schwabe $2010^{9}$ und insbesondere Schwabe $2012^{10}$ erforschen, wie ein Internetangebot, nämlich die regionalgeschichtliche Website www.vimu.info genutzt wird. Schwabe nahm bspw. das Nutzerverhalten in den Blick und konnte verschiedene Nutzertypen unterscheiden und deren Potentiale für historisches Lernen bestimmen. Auch die empirischen Untersuchungen von Schmitt/Kowski $2011^{11}$ und

6 Vgl. Bettina Alavi: Wie lernen Schüler/innen mit historischer Selbstlernsoftware? In: Judith Martin/Christoph Hamann: Geschichte, Friedensgeschichte, Lebensgeschichte. Herbolzheim 2007. S. 205-220.

7 Vgl. Bettina Alavi/Marcel Schäfer: Historisches Lernen und Lernstrategien von Jugendlichen. Eine empirische Untersuchung zu historischer Selbstlernsoftware. In: Bettina Alavi (Hrsg.): Historisches Lernen im virtuellen Medium. Heidelberg 2010. S. 75-93.

8 Eine erste Studie dieser Art war Michele Barricelli/Ruth Benrath: „Cyberhistory“. Studierende, Schüler und Neue Medien im Blick empirischer Forschung. In: Geschichte in Wissenschaft und Unterricht 54 (2003), S. 337-353. Hier wurde die Nutzung einer „Infotainment-CD-ROM“ zur Industrialisierung durch ältere Schüler/innen untersucht. Da es sich um eine sehr kleine Studie handelt, die bereits mehrere Jahre zurückliegt, bleibt diese im Folgenden unberücksichtigt.

9 Vgl. Uwe Danker/Astrid Schwabe: Zielloses Wandern im ,Cyberspace‘ oder autonomes Lernen im virtuellen Raum? Ein empirischer Werkstattbericht zur (außer-)schulischen Nutzung des regionalhistorischen Virtuellen Museums www.vimu.info. In: Bettina Alavi (Hrsg.): Historisches Lernen im virtuellen Medium. Heidelberg 2010, S. 95-130.

10 Vgl. Astrid Schwabe: Historisches Lernen im World Wide Web: Suchen, flanieren oder forschen? Fachdidaktisch-mediale Konzeption, praktische Umsetzung und empirische Evaluation der regionalhistorischen Website Vimu.info. Göttingen 2012 (Beihefte zur Zeitschrift für Geschichtsdidaktik. Bd. 4). Vgl. ihre Ausführungen in diesem Band zu genaueren Informationen über ihre Forschungen.

11 Vgl. Christine Schmitt/Nicola Kowski: Zwischen Handbuch und „Facebook“. Was erwarten Studierende von einem geschichtlichen Fachportal? Online-Umfrage zu historicum.net Geschichtswissenschaften im Internet. In: Geschichte in Wissenschaft und Unterricht 62, (2011), H.11/12, S. 655-668. 
John $2013^{12}$ widmen sich dem Lernen an digitalen Medien. Schmitt/Kowski untersuchten die Nutzung von www.historicum.net durch Geschichtsstudierende, während John 2013 die Nutzung ihrer onlinebasierten geschichtsdidaktischen Einführungsveranstaltung bei ihren Studierenden erfragt. Bei letzterem spielt auch das Lernen mit digitalen Medien eine Rolle, wenn erfragt wird, ob die Studierenden im Wiki der Veranstaltung kommunizieren oder die Blog-Funktion nutzen.

Dem Lernen im digitalen Medium widmete sich Hodel 2010.13 Er untersuchte, wie sich Jugendliche im Rahmen schulischen Geschichtsunterrichts selbständig Informationen, z.B. für ein Referat beschaffen, untersuchte also die im Anfangsbeispiel aufgegriffene Situation, in der ein Schüler zu „Amsterdam“ recherchierte. Hodel ging es dabei einerseits um die Nutzung von Mediensystemen für die Recherche, zum anderen aber auch um die Verarbeitung der Online zugänglichen Inhalte für ein schriftliches Referat zu einem historischen Thema. Er fand heraus, dass Schüler/innen das Internet als Nachschlagewerk nutzen und dabei Textstellen bevorzugen, die frei von historischen Deutungsmustern sind, also nach ihrer Ansicht „neutrales Wissen“ enthalten. Dieses Wissen nehmen sie als Allgemeingut wahr, das lediglich gesammelt und in eine gut rezipierbare Form gebracht werden muss. Dazu werden die Textstellen kopiert und nach zwei Mustern zusammengefügt:

1. Mehrere unterschiedliche, meist unbenannte Textstellen werden mit kurzen eigenen Einschüben wie in einem Mosaik zusammengefügt.

2. Unterschiedliche Elemente wie eine Zeitleiste, ein Wikipedia-Artikel, Bilder werden aneinandergehängt und als Basisinformation zu einem mündlichen Vortrag genutzt.

Hodel fragt kritisch, ob der Typ 1 (Mosaik) nicht ein neuer, digitaler Typus einer historischen Erzählform darstellt, da ein erfolgreiches Plagiat (und das wäre Typ 1) eine Bestimmung der Triftigkeit des Ausgangstextes und damit historisches Denken voraussetzt.

Betrachtet man die vorliegenden wenigen empirischen Untersuchungen insgesamt, so fällt auf, dass diese vorrangig das Lernen an digitalen Medien erfor-

12 Vgl. Anke John, Anke: Geschichtsdidaktik 2.0. Vom Nutzen und Nachteil internetbasierten Studierens im Lehramt Geschichte. In: Susanne Popp u.a. (Hrsg.): Zur Professionalisierung von Geschichtslehrerinnen und Geschichtslehrern. Göttingen 2013 (Beihefte zur Zeitschrift für Geschichtsdidaktik. Bd. 3) S. 279-298.

13 Vgl. Jan Hodel: Geschichtslernen mit Copy and Share. In: Bettina Alavi (Hrsg.): Historisches Lernen im virtuellen Medium. Heidelberg 2010, S. 111-130. Vgl. Seine Ausführungen in diesem Band für genauere Informationen über seine Forschungen. 
schen. Nur eine Untersuchung liegt zum Lernen im digitalen Medium vor. Das Lernen mit digitalen Medien wird gestreift; keine Untersuchung widmet sich aber intensiv und allein diesem Modus. Zum Lernen über digitale Medien liegt keine empirische Untersuchung vor, obwohl auch da internationale Studien über die weltweite Verständlichkeit von „historischen“ Webseiten denkbar wären.

Strukturiert man die vorliegenden empirischen Untersuchungen nach methodischen Gesichtspunkten, so findet man quantitative Studien, wie die von Schwabe 2012 und John 2013 sowie Schmitt/Kowski 2011 sowie qualitative Studien wie die von Hodel 2010 und Alavi/Schäfer 2010. Strukturiert man weiter nach der Art der Datenerhebung so finden sich empirische Untersuchungen,

1. die die Schüler/innen direkt beobachten (Alavi 2007, Alavi/Schäfer 2010) oder aber den Entstehungsprozess ihrer Produkte untersuchen (Hodel 2010);

2. die die Studierenden per online-Fragebogen nach deren Einschätzung von digitalen Lernangeboten mit historischen Inhalten fragen (Schmitt/Kowski 2011, John 2013);

3. die digital erhobenen Nutzerdaten auswerten (Danker/Schwabe 2010, Schwabe 2012).

Die geringe Zahl empirischer Untersuchungen, die sich nur beschränkt allen vier Modi des historischen Lernens im Digitalen widmen, lässt sich möglicherweise dadurch erklären, dass es sich bei der geschichtsdidaktischen Community, die über die Auswirkungen des Digitalen Wandels auf das historische Lernen arbeitet, um einen sehr kleinen Kreis handelt - er ist mit den Autor/innen dieser Publikation schon fast erschöpft. Innerhalb dieses Kreises liegt das Interesse eher auf theoretischen Weiterentwicklungen und praktischen Anwendungen, denn auf empirische Untersuchungen. Zudem sind solche Untersuchungen zeitaufwändig und ihre Aussagekraft - gerade bei qualitativen Untersuchungen mit niedriger Fallzahl - äußerst begrenzt. Auch setzen viele Lehrkräfte digitale Medien im Geschichtsunterricht immer noch selten ein, sodass das Untersuchungsobjekt fehlt.

Im Folgenden werden die Ergebnisse von drei Studien näher betrachtet, nämlich die von Alavi 2007 und Alavi/Schäfer 2010 und die von Schmitt/Kowski 2011 sowie John 2013. Die beiden anderen genannten Studien (Hodel 2010, Schwabe 2012) sind mit Einzelbeiträgen in dieser Publikation vertreten.

\section{Erkenntnisse aus empirischen Untersuchungen}

Im Unterschied zum eingangs beschriebenen Beispiel des Umgangs mit der Website des Anne-Frank-Hauses ging es bei meinen Forschungen (Alavi 2007, Alavi/Schäfer 2010) um formelle Lernprozesse, also um eigens für die Bearbei- 
tung in den neuen Medien entwickelte Lernaufgabe mit historischem Inhalt, die von einem Schulbuchverlag noch heute als Zusatzmaterial zu einem Schulbuch angeboten wird. ${ }^{14}$ Solche CDs werden heute aber kaum noch produziert, da die Entwicklung teuer und deren Gewinn für das auf kommunikative Prozesse und einen breiten Informationszugriff angewiesene historische Lernen begrenzt ist. Eine sinnvolle historische Lernaufgabe ${ }^{15}$ für ein solch in sich geschlossenes Medium zu entwickeln ist sehr schwierig. Für Geschichte werden heute eher Moodle-Lernumgebungen oder Webquests entwickelt. Gleichwohl können viele Ergebnisse der Untersuchung darauf übertragen werden. Untersucht wurde, wie Schülerpaare einer 6. Klasse (Alavi 2007) und einer 9. Klasse (Alavi/Schäfer 2010) mit einer solchen Lernaufgabe arbeiten, welche Lernstrategien sie verfolgen und wie sie zur Lösung kommen. Dazu wurden die Schülerpaare per Videoscreening aufgenommen, deren Kommunikation transkribiert und mit der Methode des lauten Denkens analysiert. Ein anschließend verteilter Fragebogen fragte die Einschätzung der Schüler/innen bezüglich ihrer Arbeit mit der Lernaufgabe ab. Die Lernaufgabe der 6. Klasse (Realschule) bestand darin, das Giebelfeld einer Kirche mit der Abbildung des Jüngsten Gerichts in ein Schaubild $\mathrm{zu}$ abstrahieren. Die 9. Klasse (Realschule) sollte das Kriegstagebuch einer Handwerkerfrau aus dem 1. Weltkrieg mit Bildern (Foto, Plakat etc.) illustrieren und damit kontextualisieren. ${ }^{16}$ Im Folgenden bündele ich die wichtigsten Ergebnisse beider Untersuchungsabschnitte:

Bei beiden Durchgängen waren diejenigen Schülerpaare am erfolgreichsten, die eine systematische Strategie der Aufgabenbearbeitung verfolgt haben. Diese bestand darin, sich zunächst einen Überblick über die zu lösende Aufgabe zu verschaffen und sich dann besonders intensiv mit den zugrunde liegenden Quellen auseinanderzusetzen. Erfolgreiche Schülerpaare strukturierten bspw. aus der intensiven Betrachtung des Giebelfeldes heraus eine Grundsystematik, die

14 Vgl. für diese Untersuchung Zeitreise multimedial. Leipzig: Mittelalter 2005 sowie das lange 19. Jahrhundert 2006.

15 Unter einer Lernaufgabe versteht man eine lehrerseitige Vorlage, die die Lerner zu einer problemorientierten kommunikativen und/oder kognitiven Handlung auffordert. Der Schwerpunkt einer Lernaufgabe liegt auf kommunikativen Problemlöseprozessen. Vgl. für den Versuch Kriterien für eine gute Lernaufgabe zum historischen Lernen im digitalen Medium zu entwickeln Bettina Alavi/Marcel Schäfer: Elemente sinnvoller netzbasierter historischer Lernaufgaben - aufgezeigt an einem Beispiel. In: Bettina Alavi (Hrsg.): Historisches Lernen im virtuellen Medium. Heidelberg 2010. S. 239-252. Für Anregungen aus dem Fremdsprachenlernen vgl. Karin Biebighäuser/Marja Zibelius/Torben Schmidt: Aufgaben 2.0. Konzepte, Materialien und Methoden für das Fremdsprachenlehren und -lernen mit digitalen Medien. Tübingen 2012.

16 Beide Lernaufgaben wiesen unter geschichtsdidaktischen Gesichtspunkten große Schwächen auf, die hier aber nicht im Detail aufgezeigt werden können. 
sich auf das abstrahierende Schaubild übertragen ließ. Sie kommunizierten bei der Entwicklung der Grundsystematik über diese, aber auch über ihre „Theorie“, nämlich, dass die Quelle (Giebelfeld) und das Schaubild die gleiche Grundstruktur haben müssten. Ähnlich war es im zweiten Durchgang. Erfolgreiche Schülerpaare sahen sich zunächst die Bilder intensiv an und gingen diese immer wieder von Anfang an durch, um sie einem Tagebuchausschnitt zuzuordnen. Dadurch erarbeiteten sie sich ein Bildarchiv, was zu Beginn erhebliche Zeit in Anspruch nahm. Dann aber wurden die Zuordnungen immer schneller und treffsicherer. Die weniger erfolgreichen Schülerpaare hingegen waren zur Entwicklung einer solchen Strategie nicht fähig. Sie lieferten sich den Einzelheiten aus, weil sie sich keinen Gesamtüberblick über die Aufgabe verschafften, sondern sogleich Details betrachteten, die sie nicht zur Entwicklung eines systematischen Lösungswegs nutzen konnten. Das führte bspw. dazu, dass bei der Beschriftung des Schaubildes als Abstraktion des Giebelfeldes alles Mögliche ausprobiert wurde und die Lösung erst nach längerer Zeit nach dem Prinzip „Trial and Error“ gefunden werden konnte. Die Schülerpaare waren eher zur Entwicklung einer systematischen Aufgabenbearbeitung fähig, wenn sie konstruktiv mit ihren Partnern kooperierten, indem sie sich gegenseitig auf dem gleichen Stand hielten (z.B. beim Leseverständnis), sich gegenseitig etwaige Lösungswege kommunizierten und diese dann auch mit einer gewissen Hartnäckigkeit verfolgten. Fiel ein Partner etwa durch eine Konzentrationsschwäche aus, versuchte der andere diesen durch Erläuterungen wieder an die Aufgabe heranzuführen.

Dieses Ergebnis bedeutet, dass im Geschichtsunterricht ein größeres Gewicht auf die Entwicklung einer solchen Fähigkeit zur Erarbeitung einer Systematik der Aufgabenbearbeitung gelegt werden müsste. Gleichzeitig gibt es aber noch keine Typik der historischen Lernaufgaben anhand derer diese Kompetenz zielgerichtet aufgebaut werden könnte. Es ist auch noch ungeklärt, was die Spezifik von „historischen“ Lernaufgaben ausmacht (hier vielleicht die Quellengrundlage) und welche Anteile $\mathrm{zu}$ einer fachunabhängigen Kompetenz gehören. Die Abstraktion eines Bildes in ein Schaubild wäre nämlich auch in anderen Fächern denkbar, z.B. als Abstraktion des Handlungsmusters einer Kurzgeschichte in ein Schaubild.

Die Untersuchungen haben auch gezeigt, dass spezifische historische Kenntnisse die Bearbeitung der Aufgabe erleichterten bzw. sie erst ermöglicht haben. Dies waren zum einen Kenntnisse über die Spezifik einer Quellenart. Nicht allen Schüler/innen war bspw. präsent, dass ein Tagebuch chronologisch aufgebaut ist und deshalb eine Zuordnung der Bilder nach einem Jahreszahlenabgleich relativ mühelos erfolgen konnte. Auch die Kenntnis, dass das Giebelfeld ein typisches Bildprogramm des Mittelalters enthält, das die christliche Jenseitsvorstellung stilisiert, hätte den Schüler/innen einen Hinweis darauf 
gegeben, die Hauptcharakteristika dieses Bildes zu abstrahieren. Somit wäre es sinnvoll im Geschichtsunterricht gerade die Spezifik der verschiedenen Quellenarten immer wieder anzusprechen, um den Schüler/innen den Umgang mit diesen zu erleichtern. ${ }^{17}$ Hinzu kommt dass die Schüler/innen bei ihrer Bearbeitung der Lernaufgabe Verknüpfungen mit ihrem Vorwissen aus dem Geschichtsunterricht vornehmen. So kommentierten sie etwa ein Bild zum 1. Weltkrieg mit „Stellungskrieg“, obwohl dies den Hinweisen in der Selbstlernsoftware nicht zu entnehmen war. Auch methodische Kompetenzen, z.B. zur Bildanalyse (Einteilung in Bildabschnitte, Entschlüsselung von Symbolen etc.), die im Geschichtsunterricht angebahnt wurden, werden angewendet, wenn die Aufgabe dieses verlangt. Diese Interdependenzen klarer zu fassen wäre eine reizvolle Aufgabe für die weitere Forschung.

Ein weiteres deutliches Ergebnis der Untersuchung war, dass die Schüler/innen sehr ökonomisch arbeiten, d.h. nur genau das machen, was von ihnen explizit gefordert wird und was minimal zur Lösung ausreicht. Schüler/innen lesen den Inhalt (z.B. des Tagebuchs) nicht genau durch, wenn eine Schlagwortsuche ausreicht; sie schlagen unverstandene historische Begriffe nicht nach, wenn ihnen kein Glossar angeboten wird und wenn sie vermuten, dass das „Überlesen“ des Begriffs nicht der Lösung der Lernaufgabe entgegensteht. Dies bedeutet, dass die Denkoperationen, die zum Lösen der Aufgabe und damit zum historischen Lernen durchgeführt werden, vorab ganz genau überlegt werden müssen. Hier scheint mir noch eine große Entwicklungsaufgabe für Lernaufgaben zum historischen Lernen $\mathrm{zu}$ bestehen. In diesem Zusammenhang wird von den Schüler/innen explizit ein Feedback eingefordert, das ihnen Sicherheit gibt und das sie im Geschichtsunterricht von der Lehrperson gewöhnt sind. Gerade auch ein Zwischenfeedback gibt den Schüler/innen Sicherheit und vermeidet gleichzeitig ein orientierungsloses Suchen nach dem Prinzip „Trial and Error“. Generell positiv gesehen wurde von den Schüler/innen, aber auch von den Studierenden der im Folgenden dargestellten Untersuchungen, dass Lernaufgaben im digitalen Medium in der eigenen Geschwindigkeit bearbeitet werden können und die Möglichkeit besteht, sich Bestandteile immer wieder anzusehen.

Im Folgenden fasse ich die Ergebnisse der Untersuchungen von Schmitt/ Kowski 2011 wie von John 2013 pointiert zusammen: Ein Ergebnis beider Umfragen war, dass Studierende Internetangebote, sei es auf einem historischen Fachportal wie historicum.net, sei es in einer Blended Learning-Umgebung „Grund-

17 Hans-Jürgen Pandel spricht von Gattungskompetenz, die aber mehr beinhaltet als das hier Angesprochene. Vgl. Hans Jürgen Pandel: Geschichtsunterricht nach PISA. Schwalbach/Ts. 2005. 
lagen historischen Lernens“, primär dazu nutzen (wollen), um einen fachlich abgesicherten Einstieg ins Thema zu bekommen, sich thematisch zu orientieren und aktuelle weiterführende Literaturhinweise zu finden. Sie nutzen diese Internetangebote also wie ein herkömmliches Handbuch, aber eines, das überall zugänglich ist und das aktueller erscheint. Diese Angebote, die instruktiv den Erwerb deklarativen Wissens fördern, sind beliebt und werden als sinnvolle Ergänzung im Studium angesehen, auch wenn bei den Angeboten häufig eine mediengerechtere Gestaltung angemahnt wird, also z.B. ein strukturiertes Design mit Visualisierungen, Hypertextstrukturen und intertextuelle Vernetzungen. Allerdings nimmt dieses Bedürfnis nach strukturierten Themeneinstiegen mit der Studiendauer ab, an fast die gleiche Stelle treten die Möglichkeiten der Fachrecherche, die das Internet bietet. ${ }^{18}$

Dass die Fachrecherche im Internet mit zunehmender Studiendauer an Bedeutung gewinnt, könnte bedeuten, dass diese im Studium erlernt wird/erlernt werden muss und dann die einfache Google-Suche durch die gezielte und effektivere Recherche auf Fachportalen ersetzt werden kann. Aufgrund der Vielzahl und Diversität der Angebote ist die schlichte Kenntnis von Adressen und der damit verbundenen Potenziale wichtig, ähnlich dem Wissen über Handbücher, Lexika, Bibliographien, wie es traditionell im Geschichtsstudium erworben wird. Dies scheint eine fachspezifische Online-Kompetenz zu sein, die im Studium erlernt werden muss, denn wo man gewünschte Informationen zu historischen Sachverhalten schnell und zuverlässig findet, wo Quellen oder aber didaktisches Material digital zugänglich ist, das muss erlernt werden und sollte in den Kanon der im Geschichtsstudium zu erwerbenden Kompetenzen aufgenommen werden.

Als weiteres Ergebnis ergeben beide Untersuchungen, dass Studierende interaktive Elemente und Formen des gemeinschaftlichen Lernens wie das Erstellen von Wikis und die Teilnahme an Blogs nur ungern nutzen (wollen). Sie beschränken sich gerne auf die Möglichkeiten des Web1.0 und drücken sich geradezu in Lernsituationen um die interaktiven Lernangebote des Web 2.0. Die Gründe hierfür sind unklar. Die Autorinnen der genannten empirischen Untersuchungen verweisen auf die in der JIM-Studie ${ }^{19}$ festgestellte private Nutzung von Facebook und Chat-Rooms. Anscheinend besteht hier unter den Heranwachsenden und jungen Erwachsenen eine Trennung der Sphären, die noch nicht miteinander verzahnt sind. M.E. könnten Gründe für die mangelnde Partizipation bei der Erstellung von Wikis, bei Kommentaren und Blogs in Situatio-

18 Schmitt/Kowski 2011 (wie Anm. 11), S. 660.

19 D.i. die Studie Jugend, Information, (Multi-)Media, die 2012 vom Medienpädagogischen Forschungsverbund Südwest zum 15. Mal vorgelegt wurde. 
nen des historischen Lernens auch darin bestehen, dass diese sich inhaltich und formal von den privaten Anmerkungen in Form von „Wie geht's?“ oder „Dein Bericht über die Geburtstagsparty ist super!“ substantiell unterscheiden. Zum einen müssen sich die Beiträge in Form von Wikis und Blogs inhaltlich durch historisches Wissen, strukturierte Argumente und einem klarem Bezug zu einer historischen Fragestellung ausweisen, zum anderen erfordern fachliche Wikis und Blogs formale Kenntnisse, z.B. über die konzeptionelle Mündlichkeit eines Blogs ${ }^{20}$ sowie über soziale Kompetenzen, z.B. bei Überarbeitungen von Wiki-Eintragungen eines anderen. Die Verarbeitung von Wissen in einem spezifischen neuen Medium, also das spezifische Schreiben, muss erst erlernt und geübt werden; die Vorstellung, dass „Digital Natives“ diese Kompetenzen bereits beherrschen, scheint obsolet zu sein.

Leider gibt es noch keine geschichtsdidaktischen Untersuchungen über die Wirksamkeit kollaborativer und kooperativer Lernformen mithilfe dieser Formate, genauso wenig wie über die Verzahnung von digitalen Angeboten für den Erwerb deklarativen Wissens und konstruktivistisch angelegten Angeboten zum interaktiven Lernen. Auch die Scharnierstellen zwischen Präsenzphasen und Onlinephasen bei Blended Learning Angeboten, wie sie die zitierte Veranstaltung „Grundlagen historischen Lernens“ enthält, müsste genauer untersucht und dabei bestimmt werden, was sich in den unterschiedlichen Phasen wie am effektivsten gelernt wird. ${ }^{21}$ Mit den gerade genannten Leerstellen bin ich bei den Forschungsdesideraten, die noch einmal prägnant markiert werden sollen.

\section{Forschungsdesiderate}

\section{Kompetenzaufbau}

Hier müsste zum einen ausformuliert werden, welche Kompetenzen zum fachspezifischen Umgang mit den digitalen Medien gehören und dann empirisch untersucht werden, wie diese erworben werden. Dabei müssten auch in Langzeitstudien zwischen kurzfristigen und langfristigen Lerneffekten unterschieden werden. ${ }^{22}$

20 Vgl. Jan Hodel: Historische Narrationen im digitalen Zeitalter. In: Uwe Danker/Astrid Schwabe: Historisches Lernen im Internet. Geschichtsdidaktik und neue Medien. Schwalbach/Ts. 2008. S. 182- 195. Hier besonders S. 193.

21 Vgl. John 2013 (wie Anm. 12), S. 284.

22 Vgl. Barricelli/Benrath 2003 (wie Anm. 8), S. 351. 


\section{Kooperatives und kollaboratives historisches Lernen}

In welchen Lernsettings gelingt kooperatives und kollaboratives historisches Lernen? Welche interaktiven Elemente sind besonders effektiv? Dazu gehören aber auch das Betrachten von Hemmnissen und die Formulierung von Möglichkeiten, diese abzuschwächen. ${ }^{23}$

\section{Lehrerforschung}

Geschichtslehrer/innen nutzen im Unterricht - von Ausnahmen abgesehen relativ wenig digitale Medien. Häufig fühlen Sie sich den Schüler/innen unterlegen oder aber sehen keinen Zusammenhang zwischen Facebook, das ihre Schüler/innen nutzen, und der Veränderungen des Geschichtsbewusstseins und der Geschichtskultur. Hier wäre eine Lehrerforschung sinnvoll, die beispielsweise untersucht, wie Innovationen implementiert werden können.

\section{Interdisziplinäre empirische Untersuchungen}

Lernsituationen in digitalen Medien sind auch dadurch gekennzeichnet, dass Kompetenzen aus mehreren Domänen abgerufen und angewendet werden müssen. Um ein Wiki auszuführen braucht es Medienkompetenz, d.h. Wissen, wie ein Wiki aufgebaut ist und welche formalen Funktionen es hat. Daneben braucht es historische Kompetenzen, d.h. u.a. Wissen, was an historischen Inhalten gesichert und was strittig ist. Sprachliche Kompetenzen sind nötig, um den Wiki-Eintrag auszuführen und soziale Kompetenzen, wenn der Beitrag eines anderen redigiert wird. Reizvoll wäre es beispielsweise auch zu überlegen, wie Medien- und historische Kompetenzen in Unterrichtssituationen gemeinsam aufgebaut werden (müssen) und was das Fach Geschichte spezifisch zur Medienkompetenz beitragen kann.

23 Eine Anregung dazu könnte aus der Grundschuldidaktik Deutsch kommen, vgl. Natascha Naujok: Zu zweit am Computer. Eine Studie zur gemeinsamen Rezeption von Spielgeschichten im Deutschunterricht der Grundschule. München 2012. Sie beobachtete wie Grundschulkinder miteinander agierten, während sie am Computer mit Spielgeschichten beschäftigt waren. 